\title{
LES EVOLUTIONS APPORTEES AU SECTEUR DE LA SANTE A TRAVERS LA REFORME DES FINANCES PUBLIQUES EN REPUBLIQUE DEMOCRATIQUE DU CONGO DE 1998 à 2013
}

\author{
Par Joseph ARSELI MONGA MANGALA*
}

\section{Résumé}

Cet article traite des évolutions apportées au secteur de la santé à travers la réforme des finances publiques en RDC de 1998 à 2013. Il démontre qu' un Etat de droit est aussi celui où les prestations sociales procurent à la population une pleine jouissance effective de ses droits fondamentaux parmi lesquels figure le droit à la santé. Cette étude ressort l'enjeu majeur de la recherche dictée par une telle interrogation qui est celui de parvenir à se construire une appréciation des performances ou contre-performances de l'Etat au regard du renforcement, par les Finances publiques, de la jouissance effective du droit aux soins de santé de bonne qualité. Elle tire la conclusion suivant laquelle la situation actuelle renseigne que l'édification de l'Etat de droit en RDC intègre difficilement le droit à la jouissance effective des soins de santé de bonne qualité.

\section{Introduction}

La santé est l'un de secteurs les plus visibilisatoires du bon fonctionnement de l'Etat. La vie se maintien dignement si elle est en bonne santé. Les droits à la vie, à une bonne santé, et soins de santé de bonne qualité sont co-originaires. A cet égard, l'on se conviendra que la visibilisation de la responsabilité de l'Etat ne saurait être effective que sous une bonne prise en charge, par les Finances Publiques, de l'ensemble des droits fondamentaux pour la jouissance des quels les services publics dudit Etat ont raison d'exister.

En effet, l'étude de la reforme des Finances Publiques, à travers les évolutions apportées au secteur de la santé par le pouvoir public de la République démocratique du Congo, ne manquerait pas à contribuer améliorativement à la conception qu'il convient de se faire de ce qu'on appelle « Etat de Droit».

Une telle étude nous suggère de porter sur l'Etat congolais le regard qu'un consommateur porte sur un arbre fruitier. Pour un consommateur, tout arbre fruitier se juge par ses fruits, peu importe sa couleur, son feuillage, ni le parfum de son essence.

* Chef des travaux à la Faculté des Lettres et Sciences Humaines, Doctorant à la Faculté de Droit, Université de Kinshasa, Email : josepharseli@yahoo.fr. 
Par analogie, un Etat se juge être un Etat de Droit si les prestations des services publiques qui le constituent parviennent à procurer à la population une jouissance effective de ses droits fondamentaux, dont le droit à une bonne santé.

On démontrera, à ce propos que le mortel Etat de droit peut mourir aussi sous de petits coups d'écriture comptable, ${ }^{1}$ surtout lorsque ceux-ci sont la cause d'amenuisement de l'enveloppe budgétaire alloué au domaine de la santé.

La principale interrogation à laquelle la présente étude se propose de répondre se formule de la manière ci-après :

Comment se présenterait l'impact des évolutions apportées au secteur de la santé, à la suite de la réforme des Finances Publiques de 1998 à 2013 en République Démocratique du Congo?

Il ressort que l'enjeu majeur de la recherche dictée par une telle interrogation est de parvenir à se construire une appréciation, par laquelle, on indiquera, chiffres à l'appui, les performances ou contreperformances, de l'Etat au regard du renforcement, par les Finances Publiques, de la jouissance effective du droit aux soins de santé de bonne qualité. Car ce droit s'affirme comme l'une des composantes de la notion de l'Etat de droit.

Si l'Etat de droit dont se réclame la République Démocratique du Congo, ne se mesure pas uniquement par sa capacité de rendre effective la jouissance du droit aux soins de santé de bonne qualité, il n'en demeure pas moins qu'il nous soit pertinent de s'interroger si la non jouissance de ce droit ne déroge pas à l'existence d'un Etat de droit? l'Etat de droit ne serait-t-il pas infirme s'il n'intègre pas le droit aux soins de santé de bonne qualité?

Si l'Etat de droit relève d'une édification améliorativement perpétuelle des domaines essentiels de la vie, à quel niveau d'amélioration de la qualité des soins de santé de base qu'un Etat peut se proclamer être un Etat de droit? L'accès à la jouissance effective de ce droit est-il réalisable sans que l'écriture de la comptabilité budgétaire y consacre un montant à la hauteur de défis correspondants?

Les évolutions apportées au secteur de la santé primaire par la reforme des Finances Publiques de 1998 à 2013 traduisent-elles une preuve de bonne volonté politique de la par des gouvernants congolais vis-à-vis du secteur de la santé?

Telles sont des questions ramificatoires suscitées au tour de la question centrale, auxquelles nous tenterons de répondre.

La première hypothèse de réponse est une tentative d'affirmation que la reforme des Finances Publiques de 1998 à 2013 en République Démocratique du Congo, n'a pas permis à la population de jouir effectivement du droit d'accès aux soins de santé de bonne qualité comme cela était le cas pendant la brève période des premières heures post coloniales.

1 Lire la préface du Professeur BAKANDEJA, in J.M ABOLIA, Finances Publiques et comptabilité de l'Etat en République Démocratique du Congo : Principes, Pratique et perspectives, B.E.S.I.F, Kinshasa, 2005, p.5. 
La deuxième hypothèse porte à affirmer que la non jouissance du droit aux soins de santé de bonne qualité dans un Etat déroge à l'existence d'un véritable Etat de droit. Car ce droit est co-originaire au droit à la vie de bonne qualité.

La troisième hypothèse porte à affirmer que l'amélioration de la qualité des soins de santé atteint le niveau d'être qualifiée bonne si les critères des standards internationaux sont accomplis, tels que fixés par l'organisation mondiale de la santé et c'est à ce niveau que l'on peut affirmer jouir d'une des composantes incontournables d'un Etat de droit, à l'occurrence droit aux soins de santé de bonne qualité.

La quatrième hypothèse porte à affirmer que si l'écriture de la comptabilité budgétaire ne consacre pas un montant à la hauteur de défis aux soins de santé primaires de bonne qualité, l'accès à la jouissance du droit y relatif serait irréalisable.

La cinquième hypothèse porte à tenter d'affirmer que les évolutions apportées au secteur de la santé primaire, par la réforme des Finances Publiques de 1998 à 2013, ne traduisent pas une preuve de bonne volonté politique des gouvernants.

La vérification de ces cinq hypothèses nous porte à adhérer à la thèse de l'inter complémentarité méthodologique. Celle-ci nous aidera à examiner les principaux aspects de l'objet sous examen : l'exégétique, la diachronie, la descriptive, la quantitative et la qualitative seront au rendez-vous et assortis de technique documentaire.

L'inter complémentarité méthodologique aura l'avantage d'éclairer plusieurs aspects de l'objet d'étude. Ainsi, son appréhension est facilitée par un plan sommaire qui divise le travail en deux : les problèmes et évolutions apportées au secteur de la santé à travers la reforme des Finances Publiques en R.D. Congo de 1998 à 2013 sera le premier point et le deuxième aura comme intitulé les problèmes de financement du secteur de la santé au regard de l'édification d'un Etat de droit en R.D Congo.

\section{LES PROBLEMES ET EVOLUTIONS APPORTEES AU SECTEUR DE LA SANTE A TRAVERS LA REFORME DES FINANCES PUBLIQUES DE LA REPUBLIQUE DEMOCRATIQUE DU CONGO DE 1998 à 2013}

Les taux élevés de morbidité et de mortalité prématurée que connait la R.D. Congo font payer un lourd tribu au pays. Le mauvais état de santé de la population est source de peine et de souffrances; il sape l'énergie et l'aptitude de millions de congolais et les empêche à s'acquitter de leurs tâches, et de profiter de la vie. Ses conséquences économiques sont considérables. « un mauvais état de santé entrave le capital humain, amenuise le rendement de l'instruction, contrecarre les entreprises et fait obstacles à la croissance du Produit National Brut $^{2} \gg$.

Les obstacles à l'amélioration de la santé en R.D. Congo ne se limite pas à des phénomènes comme la guerre et la chute des prix des produits de base qui, pour aussi

2 Banque Mondiale, Pour une meilleure santé en Afrique, les leçons de l'expérience, la série « développement à l'œuvre », Banque Mondiale, D.C, Washington, 1995, p.1. 
bouleversants qu'ils soient, sont néanmoins temporaires. Si ces facteurs étaient les seuls en cause, la R.DCongo aurait pu réaliser davantage de progrès en vue d'améliorer la santé de sa population.

Parmi les obstacles, le plus important tient au fait que le système politique de gouvernance se révèle moins efficace, faute de bonne volonté politique, à améliorer la santé de la population. Cela se traduit par le manque d'une bonne et rigoureuse impulsion sur la gestion de budget alloué à la santé.

On constate que depuis l'an 2000 jusqu'à l'an 2013, le financement par l'Etat du système sanitaire en R.D. Congo n'est pas à la hauteur des défis qui le guettent.

\section{PROBLEMES LIES AUX MOYENS D'ACTION FINANCIERS DU SYSTEME SANITAIRE EN REPUBLIQUE DEMOCRATIQUE DU CONGO}

Les moyens d'action financiers de l'Etat constitue un domaine qui couvre deux grandes activités à savoir : les activités d'exécution des recettes et les celles d'exécution des dépenses. Il va sans dire que c'est par ces dernières que l'Etat prend en charge le financement du secteur de la santé.

L'analyse du financement du système sanitaire en République Démocratique du Congo touche de plein fouet la problématique de la mise en œuvre des moyens d'action financiers dans ce secteur. La particularité d'une telle analyse dévoile, plus spécialement une distorsions, au cours de la dernière décennie, de plus en plus croissante qui résume la problématique entre, d'une part, les charges réelles de l'Etat, incompressibles, toujours croissantes ainsi que les dépenses budgétairement prévues et celles effectivement exécutées et, d'autre part, les ressources publiques, variables dans ledit secteur, au gré des conjonctures politiques et économiques, ainsi que les recettes prévues puis celles finalement mobilisées. ${ }^{3}$

A interpréter l'article 16 de la loi financière, les charges publiques peuvent être définies comme l'ensemble des besoins identifiables qu'exprime la nécessaire viabilité de la collectivité nationale pour son équilibre économique et social. ${ }^{4}$ Les charges inventoriées et prises en compte par l'autorité budgétaire deviennent des prévisions budgétaires de dépenses; tandis que celles qui sont effectivement exécutées et prises en compte s'appellent des dépenses publiques. $^{5}$

Par ailleurs, les ressources publiques sont les potentialités financières dont dispose l'Etat, susceptibles d'être mobilisés pour faire face aux charges publiques. Les prévisions budgétaires de recettes sont les ressources à maximiser inscrites au budget qui, une fois mobilisées, deviennent des recettes publiques. ${ }^{6}$

3 Robert UMBA -DI- NDANGI, Finances Publiques, commentaires de Principes - Procédures - Pratiques des origines à nos jours en RDC, Kinshasa, B.E.C.I.F., 2006, p. 143.

4 Idem.

5 Robert UMBA -DI- NDANGI, Finances Publiques, commentaires de Principes - Procédures - Pratiques des origines à nos jours en RDC, Kinshasa, B.E.C.I.F., 2006, p. 143.

6 Idem. 
a. Problèmes et évolutions du financement du secteur de la santé

Le financement du secteur de la santé en République Démocratique du Congo, l'expression de la mise en œuvre des moyens d'action financiers de l'Etat dans ledit secteur, repose principalement sur quatre sources comprenant des sources financières domestiques (budget de l'Etat provenant du Gouvernement central et provincial ou des entités territoriales décentralisées); du financement communautaire (paiement direct des ménages et la participation communautaire); du financement privé (lucratif ou non lucratif); et des ressources financières qui proviennent de l'aide internationale de fonds spéciaux et des mutuelles. ${ }^{7}$ On constate que tous ces sources n'évoluent pas sans problèmes. Nous y reviendrons.

Après la décennie quatre vingt, malgré le déclin général du financement public, le système sanitaire a fonctionné assez valablement jusqu'au début des années nonante (quatre vingt dix), période à partir de laquelle, le pays a connu des crises multiformes, provoquant une compression drastique des financements publics.

Le financement extérieur et les recettes du recouvrement des coûts ont permis le maintien d'une offre minimale des services de santé; mais de faible qualité. ${ }^{8}$

Cette situation de désengagement de l'Etat dans le financement du secteur de la santé depuis 1987 a conduit à la perte progressive du leadership de l'Etat. Car les droits à la vie et aux soins de santé de bonne qualité en pâtissaient déjà. Tout ceci se faisait dans le dysfonctionnement du système de gestion budgétaire qui, depuis de nombreuses années a en croire le Professeur MABI MULUMBA, ${ }^{9}$ encore Président de la Cour des comptes en R.D. Congo,, s'explique pour une grande part par l'absence du vote de loi portant arrêt des comptes et règlement définitif du budget de l'Etat. En d'autres termes, l'exécution de différents budgets se faisaient dans l'inconformité au principe de redevabilité. Elle a consacrée, ajoute t-il, dans ses germes, l'impunité, d'où de graves dérives constatées : des dépassements dans la consommation des crédits, l'exécution des dépenses non prévues au budget au détriment de celle prévues. ${ }^{10}$

C'est à partir de l'année 2000 que l'on commence à noter quelques changements dans la part du budget de l'Etat alloué à la santé. Mais ces allocations sont demeurées insuffisantes par rapport aux besoins réels et aux engagements internationaux. ${ }^{11}$

b. Problèmes et évolutions liés au budget consacré à la santé

Les problèmes que pose le budget de l'Etat consacré à la santé sont de cinq ordres, à savoir :

7 Lire le Plan National de développement sanitaire (PNDS) 2011 - 2015 de la République Démocratique du Congo, Ministère de la Santé publique, Secrétariat général, Kinshasa, 2010, p. 47.

8 Lire le Plan National de développement sanitaire (PNDS), 2011 -2015, op. cit, p. 47.

9 MABI MULUMBA, Préface, In R. UMBA - DI - NDANGI, op. cit.; p3.

10 Idem.

11 Lire le Plan National de développement sanitaire(PNDS), 2011 -2015, op. cit, p. 48. 
1. Sa modicité (faible en volume) par rapport aux besoins et aux engagements internationaux;

2. Son taux d'exécution est également faible;

3. Son affectation ne prend pas toujours en compte les priorités du secteur.

En effet, le budget de l'Etat consacré à la santé est affecté en dehors de tout processus de priorisation relevant du secteur. ${ }^{12}$

Exemplativement, si nous prenons la zone de santé comme unité opérationnelle de la politique nationale de santé, on constate qu'elle n'est pas prise en compte dans le budget de l'Etat consacré à la santé;

1. Le ministre de la santé n'est pas associé dans la fixation des enveloppes, l'élaboration du plan d'engagement ainsi que du plan de trésorerie. Ces documents lui sont communiqués par le ministre du budget qui en a la prérogative conformément aux textes fixant les attributions des ministères. ${ }^{13}$

2. La passation des marchés publics étant centralisée à la commission d'adjudication qui siège au ministère du budget.

En considérant ces problèmes décrits, il y a lieu de se convaincre que le ministre de la santé éprouve certainement des difficultés pour assurer la qualité des biens et services qui sont acquis sur fonds domestiques d'une part et d'autre part, il subit de façon passive des répercussions liées à de longues procédures sur lesquelles il n'a aucun pouvoir.

Ci-contre le tableau qui illustre la mise en œuvre des moyens d'action financiers 1998 à 2013 pour le secteur de la santé :

12 Lire le plan National de développement sanitaire (PNDS), 2011 - 2015, op. cit, p. 48.

13 Idem. 


\begin{tabular}{|c|c|c|c|c|c|c|c|c|c|c|c|c|c|c|c|c|c|c|}
\hline & 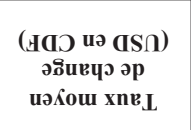 & & & & & 㐫 & ळे & $\overrightarrow{\mathcal{F}}$ & 守 & i̊ & i & : & $\frac{0}{\infty}$ & $\stackrel{\circ}{n}$ & $\frac{0}{0}$ & 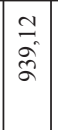 & $\frac{d}{2}$ & 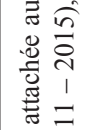 \\
\hline & 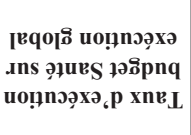 & $\begin{array}{l}\stackrel{\circ}{2} \\
\text { ñ } \\
0\end{array}$ & $\stackrel{\circ}{\vec{\sigma}}$ & 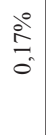 & 啇 & $\begin{array}{l}\stackrel{0}{0} \\
\infty \\
0 \\
0\end{array}$ & \begin{tabular}{|l|} 
ڤें \\
ते \\
n்
\end{tabular} & $\begin{array}{l}\stackrel{\circ}{\circ} \\
\dot{v} \\
\text { vi }\end{array}$ & $\begin{array}{l}0 \\
0 \\
\infty \\
i \\
m \\
m\end{array}$ & $\begin{array}{l}0 \\
\dot{0} \\
\sim \\
m \\
m\end{array}$ & $\begin{array}{l}\stackrel{\circ}{2} \\
\text { in }\end{array}$ & $\begin{array}{l}\infty \\
\infty \\
\infty \\
\infty\end{array}$ & 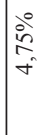 & 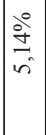 & $\stackrel{\circ}{\stackrel{\circ}{*}}$ & & & 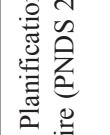 \\
\hline & 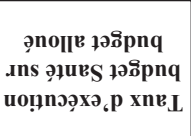 & ڤे & $\stackrel{8}{\circ}$ & 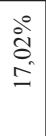 & $\begin{array}{l}0 \\
8 \\
0 \\
0 \\
\text { in } \\
\text { n. }\end{array}$ & $\begin{array}{l}\stackrel{0}{a} \\
2 \\
2\end{array}$ & $\begin{array}{l}0 \\
i \\
n \\
n \\
n \\
n\end{array}$ & $\begin{array}{l}\stackrel{0}{ } \\
\text { bे } \\
\text { ले }\end{array}$ & $\begin{array}{l}\stackrel{0}{2} \\
i \\
i \\
i n\end{array}$ & $\begin{array}{l}0 \\
\vdots \\
0 \\
\dot{f}\end{array}$ & $\begin{array}{l}\stackrel{0}{0} \\
\infty \\
\infty \\
0 \\
0\end{array}$ & $\begin{array}{l}0 \\
0 \\
0 \\
2 \\
2\end{array}$ & लें & 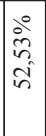 & 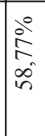 & 㝘 & 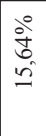 & 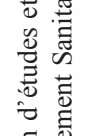 \\
\hline & 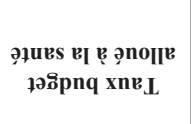 & $\stackrel{\circ}{\infty}$ & $\stackrel{\circ}{\circ}$ & $\begin{array}{l}0 \\
\vdots \\
\hat{\sigma} \\
0\end{array}$ & $\stackrel{\leftrightarrow}{\alpha}$ & $\begin{array}{l}0 \\
0 \\
\infty \\
0 \\
0\end{array}$ & 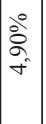 & $\begin{array}{l}\stackrel{0}{0} \\
\dot{\sigma} \\
\text { in }\end{array}$ & 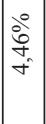 & $\begin{array}{l}\stackrel{0}{0} \\
\dot{\sigma} \\
\dot{+}\end{array}$ & $\begin{array}{l}\text { तें } \\
\text { ले }\end{array}$ & $\begin{array}{l}\text { ¿ें } \\
\text { ले } \\
\text { ले }\end{array}$ & $\begin{array}{l}\stackrel{0}{\grave{\lambda}} \\
\text { in }\end{array}$ & $\begin{array}{l}\therefore \\
i \\
\infty \\
i n\end{array}$ & $\begin{array}{l}\stackrel{0}{2} \\
\dot{+} \\
\dot{m}\end{array}$ & 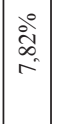 & $\begin{array}{l}\stackrel{0}{2} \\
i \\
\dot{\gamma} \\
+\end{array}$ & 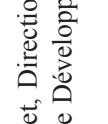 \\
\hline ช్ & 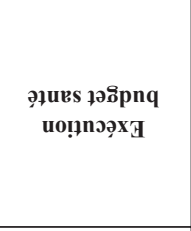 & 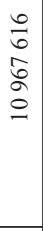 & 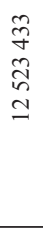 & $\begin{array}{l}8 \\
8 \\
\circ \\
8 \\
\circ \\
\dot{q}\end{array}$ & 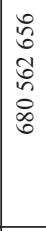 & $\begin{array}{l}n \\
2 \\
n \\
n \\
n \\
\infty \\
\infty \\
m\end{array}$ & 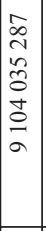 & 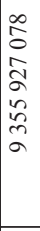 & 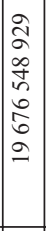 & 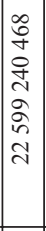 & $\begin{array}{l}0 \\
\infty \\
0 \\
\infty \\
0 \\
0 \\
\infty \\
\infty \\
2 \\
i n \\
i n\end{array}$ & $\begin{array}{l}\text { J } \\
0 \\
2 \\
2 \\
2 \\
6 \\
6 \\
n \\
n\end{array}$ & 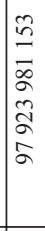 & 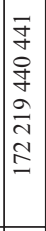 & $\begin{array}{l}0 \\
0 \\
n \\
n \\
n \\
n \\
7 \\
n \\
m\end{array}$ & 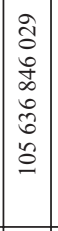 & 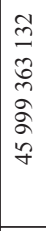 & 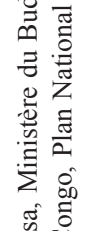 \\
\hline & วุurS મəถึpng & 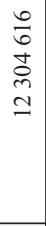 & 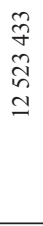 & 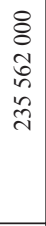 & 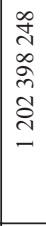 & \begin{tabular}{c}
$\infty$ \\
0 \\
$n$ \\
$i$ \\
$i n$ \\
\multirow{2}{*}{} \\
0 \\
-
\end{tabular} & 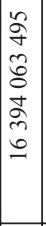 & 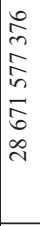 & 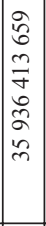 & $\begin{array}{l}0 \\
\tilde{N} \\
0 \\
0 \\
0 \\
\infty \\
0 \\
\infty \\
F\end{array}$ & 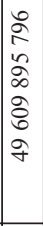 & $\begin{array}{l}\infty \\
2 \\
2 \\
2 \\
2 \\
2 \\
2 \\
\tilde{1} \\
n\end{array}$ & 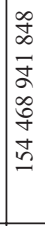 & 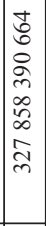 & $\begin{array}{l}\vec{N} \\
\hat{N} \\
\infty \\
\tilde{n} \\
0 \\
\infty \\
\cdots \\
\tilde{N}\end{array}$ & 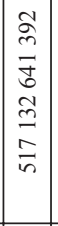 & 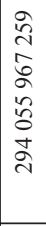 & 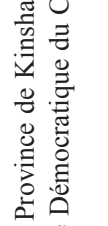 \\
\hline \multirow{3}{*}{$\begin{array}{l}\overline{0} \\
\text { के }\end{array}$} & 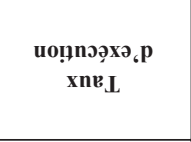 & $\begin{array}{l}\circ \\
\vdots \\
\vdots \\
\dot{0} \\
\dot{0}\end{array}$ & $\begin{array}{l}\stackrel{\circ}{\circ} \\
\stackrel{\circ}{\circ}\end{array}$ & $\begin{array}{l}\circ \\
\stackrel{0}{2} \\
\tilde{n}\end{array}$ & 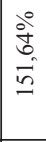 & 高 & $\begin{array}{l}0 \\
\frac{0}{0} \\
\dot{0} \\
+\infty \\
0\end{array}$ & $\begin{array}{l}0 \\
8 \\
0 \\
i \\
i\end{array}$ & 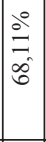 & $\begin{array}{l}0 \\
\vdots \\
0 \\
0 \\
0 \\
0\end{array}$ & 守 & 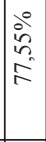 & 卞 & 总 & $\begin{array}{l}\circ \\
\hat{n} \\
\hat{n} \\
\hat{q}\end{array}$ & $\mid \begin{array}{l}0 \\
8 \\
8 \\
0 \\
0\end{array}$ & $\begin{array}{l}0 \\
\stackrel{0}{0} \\
0 \\
0\end{array}$ & 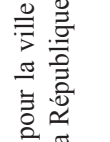 \\
\hline & 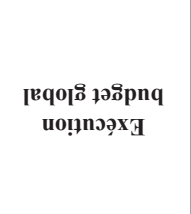 & 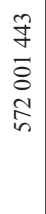 & 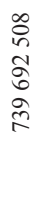 & 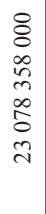 & $\begin{array}{l}\infty \\
0 \\
0 \\
0 \\
0 \\
0 \\
0 \\
0 \\
1 \\
1 \\
0\end{array}$ & 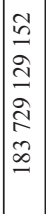 & 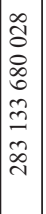 & 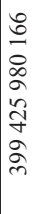 & 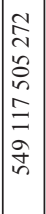 & 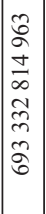 & $\begin{array}{l}\infty \\
0 \\
\infty \\
\infty \\
\hat{b} \\
2 \\
\cdots \\
0 \\
0 \\
\sigma\end{array}$ & 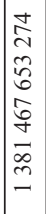 & 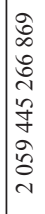 & 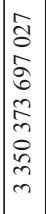 & 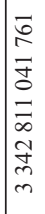 & & & 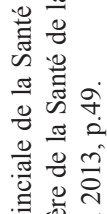 \\
\hline & [вq0|§̊ ๆəôpng & $\begin{array}{l}n \\
0 \\
+ \\
\infty \\
\infty \\
\infty \\
\infty \\
0\end{array}$ & $\begin{array}{l}\infty \\
0 \\
n \\
\text { aे } \\
\text { ले } \\
\text { r }\end{array}$ & 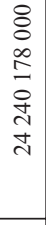 & 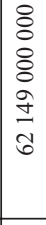 & 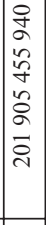 & 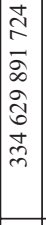 & $\begin{array}{l}8 \\
0 \\
0 \\
8 \\
0 \\
m \\
0 \\
0 \\
\infty \\
i \\
\text { n. }\end{array}$ & 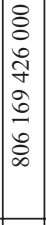 & \begin{tabular}{|l|}
0 \\
0 \\
0 \\
8 \\
0 \\
$\vdots$ \\
0 \\
2 \\
2 \\
0 \\
0
\end{tabular} & $\begin{array}{l}0 \\
0 \\
0 \\
0 \\
0 \\
0 \\
0 \\
0 \\
0 \\
p \\
0 \\
m\end{array}$ & 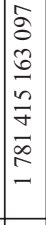 & 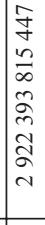 & $\mid \begin{array}{l}n \\
2 \\
0 \\
0 \\
0 \\
n \\
n \\
n \\
0 \\
0 \\
n \\
n\end{array}$ & 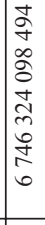 & 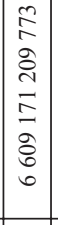 & 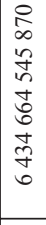 & 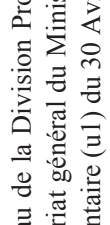 \\
\hline & วə̣uиу & 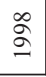 & ळे & ¿े & ठิ & ¿̊̀ & ڤ̊̀े & 商 & 哭 & ¿̊̀ & సิ & $\stackrel{\infty}{\stackrel{N}{े}}$ & ¿ें & $\stackrel{\circ}{\stackrel{2}{*}}$ & $\overline{\stackrel{\text { Dे}}{ }}$ & ì & $\stackrel{m}{\stackrel{n}{i}}$ & * \\
\hline
\end{tabular}


On note sur ce tableau que l'enveloppe du budget de l'Etat consacré à la santé a constamment évolué en valeur absolue, passant de 12304616 dollars (USD) en 1998 à 294055967259 dollars (USD) en 2013. Cependant, en pourcentage du budget global de l'Etat exercice 2011, il est resté inférieur aux engagements, internationaux notamment ceux d'Abuja ( $15 \%$ du budget de l'Etat doivent être consacrés à la santé). Le pourcentage le plus élevé du budget de l'Etat consacré à la santé à été enregistré en 2012 (7,82\%). Tandis que le pourcentage le plus élevé d'exécution du budget alloué à la santé a été enregistré en 2007 $(108,83 \%)$.

En pourcentage du produit intérieur brut, le niveau des dépenses publiques de santé est passé de $0,41 \%$ en 2000 à 1,99\% en 2009 tout en restant inférieur à $2 \%$ sur l'ensemble de la période considérée. Toutefois, ces dépenses ont évoluées proportionnellement à la part du budget de l'Etat alloué à la santé pour la même période tel que le montre la figure cidessous.

Voici l'évolution comparée du budget de la santé en pourcentage du Produit Intérieur Brut et du pourcentage du Budget de l'Etat alloué à la santé de 2000 à 2009. ${ }^{14}$

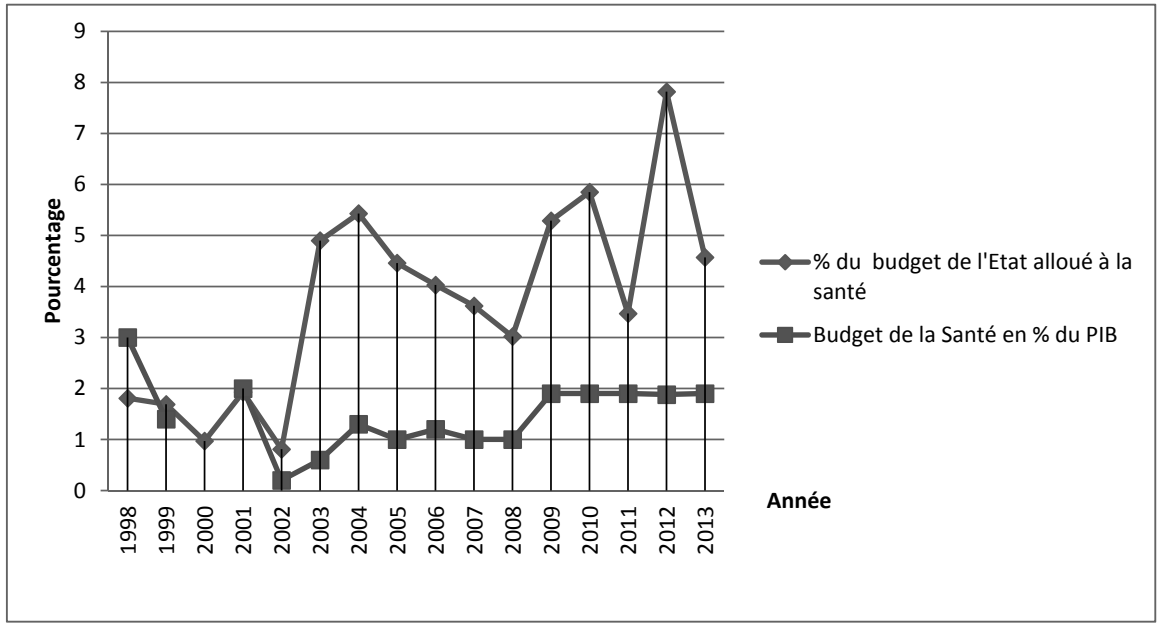

Il va sans dire que hormis les deux premières années le taux d'exécution du budget de la santé est en moyenne de 70\% au cours de la période de 1998 à 2011 avec une performance exceptionnelle observée au cours des années 2007 et 2008. Le taux d'exécution supérieur au budget alloué est la résultante des dépassements liés aux dépenses relatives aux équipements, aux urgences (épidémies) et catastrophes et, évidemment aux réajustement en cours

14 Bureau de la Division provinciale de la santé pour la province de Kinshasa, Ministère du Budget et DEP, Ministère de la Santé in Plan National de Développement sanitaire (PNDS 2011 - 2015), secrétariat général du Ministère de la Santé de la République Démocratique du Congo, Kinshasa 2010 , p. 50 . 
d'exercice budgétaire de la rémunération du personnel (salaires et primes). Le graphique suivant montre l'évolution du taux d'exécution du budget de l'Etat consacré à la santé entre 1998 et 2013. On constate que ce taux a évolué en dents de scie d'une année à une autre.

\section{PROBLEMES ET EVOLUTIONS DU PROCESSUS BUDGETAIRE DU SECTEUR DE LA SANTE}

Le processus budgétaire du secteur de la santé a peu de crédibilité dans les provinces, depuis son élaboration jusqu'à son exécution. A part les fonds de rémunérations qui sont transférés vers les provinces, les décisions concernant l'exécution des autres dépenses (principalement les fournitures équipements, construction ou réfection des bâtiments) sont prises à Kinshasa, en dépit de l'existence des gouvernements provinciaux. Très peu de fonds destinés au fonctionnement sont transférés aux provinces. Il faut ajouter à cela que les informations sur la comptabilité budgétaire ainsi que l'exécution des actes budgétaires ne sont guère communiqués régulièrement. Les districts et zones de santé sont également victimes de ce déficit. ${ }^{15}$

\section{a. Problèmes et évolutions des dépenses publiques du secteur de la santé}

Les évolutions chiffrées apportées au secteur de la santé au travers les finances publiques ne cessent de dénoter un déséquilibre entre la ville province de Kinshasa et l'arrière pays. La répartition géographique du budget est nettement en faveur de Kinshasa la capitale.

Les données sur le budget du secteur de la santé de 2002 montrent que 10 à 20 cents dollars (USD) par habitant sont allouées aux provinces, à comparer avec 1 dollar (USD) par habitants pour la ville de Kinshasa. En ce qui concerne les dépenses réelles : $60 \%$ à $65 \%$ du montant des salaires (budget exécuté) sont dépensés à Kinshasa où vit environ 10\% de l'ensemble de la population congolaise, tandis que $35 \%$ à $40 \%$ sont destinés au personnel de toutes les provinces confondues.

En 2005, 17\% de l'enveloppe budgétaire allouée aux dépenses de fonctionnement des administrations de santé des provinces et des districts a été engagé, tandis qu'en 2006, le taux d'exécution a été de 3\%. A titre illustratif, dans la province de l'Equateur, les montants de fonctionnement reçus se chiffrent) 3000 USD en 2005 et à 1500 USD en $2006 .{ }^{16}$ Le résultat final est que les administrations provinciales de la santé n'ont aucune motivation pour participer au processus d'élaboration du budget national.

Au demeurant, il y a lieu de se convaincre que ces problèmes, tels qu'ils viennent d'être dépeints, retardent l'avenue de l'offre et l'utilisation des services de santé de bonne qualité tant attendu à tous les niveaux. Ils entraînent irrévocablement, le délabrement des infrastructures et des équipements, la démotivation du personnel, la commercialisation des soins

15 Ministère de la santé Publique et Banque Mondiale, Revue des dépenses publiques, 2007.

16 Ministère de la santé Publique et Banque Mondiale, Revue des dépenses publiques, 2007. 
de santé, la rupture fréquente des stocks en médicaments essentiels, la faible utilisation des services curatifs et l'exclusion de la majeure partie de la population des services et soins de santé. Pourtant ces services publics devraient, par un bon fonctionnement, rendre effectif le droit à une bonne santé pour tous. ${ }^{17}$

b. Quelques conséquences de l'évolution des dépenses publiques du secteur de la santé

A cause du désengagements progressif de l'Etat dans le financement du système de santé, il s'est crée des flux financières où l'argent de la contribution des malades remonte pour financer les niveaux supérieurs des structures administratives. Cette pratique s'est intensifiée à partir des années 1990, lorsque les centres de santé et hôpitaux ont été amenés à reverser 5 à 10\% de leurs entrées financières aux bureaux centraux des zones de santé (BCZS) qui, eux-mêmes, devaient en faire autant pour le niveau provincial, et celui-ci pour le niveau central. ${ }^{18}$

Une telle chaine de versements servent à la réalisation des activités, mais également aux rémunérations des personnels à ces différents niveaux. Au fil des années ce système de taxation informelle et inéquitable s'est enraciné et devenu un des moteurs du système pour la survie financières des individus et des institutions.

Ce système de « survie » est également alimenté par toute une série de canaux comprenant notamment toutes sortes d'autorisation du fonctionnement ou d'ouverture des structures de santé privées, les amendes et taxes, ...; un privé à but lucratif non régulé s'est ainsi installé.

Dans un tel contexte d'absence des mécanismes adéquats pour une bonne qualité des soins, le développement anarchique du privé constitue un facteur gangreneur pour la santé de la population et un facteur d'explosion des coûts des soins de santé et partant de l'accentuation de la pauvreté (les grands contributeurs étant les ménages). Et pourtant au titre II,${ }^{19}$ où la Constitution a garanti les droits humains,...; le droit à la santé est cité nommément. ${ }^{20}$ En outre, il convient de préciser qu'à cause de la sacralité de la personne humaine, l'Etat a l'obligation de la respecter et de la protéger, ${ }^{21}$ dans le cas de figure, au travers une bonne prestation des services de la santé publique.

17 Lire le plan National de développement sanitaire, p.48.

18 Lire le plan National de développement sanitaire (PNDS), 2011 - 2015, op. cit, p. 48.

19 Constitution de la République Démocratique du Congo, Journal Officiel de la République Démocratique du Congo, $47^{\mathrm{e}}$ année, numéro spécial du 18 février 2006, p. 13.

20 Article 47, constitution de la République Démocratique du Congo, p. 23.

21 Nous estimons que « le droit à la santé (...) doit être complété par le droit à la jouissance d'une bonne prestation des soins de santé de bonne qualité. 


\section{LES PROBLEMES DE FINANCEMENT DU SECTEUR DE LA SANTE ET L'EDIFICATION D'UN ETAT DE DROIT EN REPUBLIQUE DEMOCRATIQUE DU CONGO.}

Le financement du secteur de la santé, en République Démocratique du Congo, se fait essentiellement à partir des quartes canaux : Budget de l'Etat; l'aide internationale; financement communautaire et financement privé.

Il s'avère cependant que les problèmes qui se pose dans ces canaux constituent, en tout état de cause, des entraves certaines pour la jouissance effective du droit aux soins de santé de bonne qualité. Ce droit, comme nous l'avons déjà évoqué plus haut, est pourtant l'un des droits les plus fondamentaux dont le défaut de jouissance effective s'oppose à l'exercice des libertés fondamentales et peut même offusquer l'accomplissement de certains devoirs citoyens que la Constitution rend, pourtant obligatoire. Un Etat de droit sans l'accomplissement des devoirs citoyens constitutionnellement obligatoires est indigeste.

Pour le bon fonctionnement des services publics de l'Etat, il convient que le budget puisse en servir d'instrument le plus privilégié.

Comme instrument politique, économique et juridique en vue d'améliorer le social dans ses aspects multiformes, le budget alloué à la santé, au regard des différents défis de ce secteur, constitue, au fil du temps, plus un problème qu'une solution.

\section{LES PROBLEMES CONSECUTIFS AU BIDGET CONSACRE A LA SANTE ET L'EDIFICATION D'UN ETAT DE DROIT EN REPUBLIQUE DEMOCRATIQUE DU CONGO.}

Dans tous les pays du monde, il n'y a pas de finances publiques prospères sans budget. En d'autres termes, l'on ne peut envisager la gestion des grands ensembles sans une approche prospective. $^{22}$

L'une des fonctions de l'Etat, qui mérite être la plus organisée est la fonction financière. Elle consiste, pour le gouvernement, de disposer d'un programme chiffré tant en recettes qu'en dépenses comprenant des actions à mener afin d'améliorer la situation non seulement économique, mais surtout social du pays. ${ }^{23}$ Ce programme annuel appelé budget doit viser non seulement la rationalité et l'efficacité pour éviter le gaspillage des moyens partant de l'élaboration, l'exécution et le contrôle, mais aussi et surtout « le raisonnable » dans la répartition de montants à allouer à chaque secteur. Car il ya de secteurs dont la minoration d'enveloppe budgétaire complique foncièrement l'édification de l'Etat de droit ou donne lieu à l'avènement étapiste d'un Etat de droit infirme. Il s'agit des secteurs de la santé, de l'éducation et de l'Armée.

On ne ferait pas fausse route d'affirmer à l'issu de cette analyse, que le processus d'édification d'un Etat de droit évolue décrescendo à la suite non seulement de falsification

22 J.M. ABOLIA, op. cit., p.17.

23 Idem, p. 16. 
tricheresse de l'écriture de la comptabilité budgétaire, détournement et fictivisation des dépenses, ..., mais aussi et surtout consécutivement au défaut d'une bonne preuve de bonne volonté politique au sommet de l'Etat.

a. Le processus d'édification de l'Etat de droit à l'épreuve de l'évolution du budget de la santé en République Démocratique du Congo

Si le vote du budget est une chose, son exécution en est une autre ${ }^{24}$; il convient d'ajouter ici que la raisonnable répartition de montants à chaque secteur au regard de l'édification de l'Etat de droit constitue également une chose à part entière.

Par ailleurs, il s'avère pertinent de se convaincre que la vitalité d'une nation, dans l'édification d'un Etat de droit, est fonction de la capacité dont dispose le pouvoir public d'assurer améliorativement, de manière continue, le bien-être de sa population. Cette vitalité est sujet à caution si la jouissance effective du droit aux soins de santé de bonne qualité est loin d'être une réalité vivante.

La république Démocratique du Congo est proclamé un Etat de droit,... et social, ${ }^{25}$ mais les renseignements chiffrés de la comptabilité budgétaire permet de constater une preuve de faible degré de la bonne volonté politique. Un tel sort fait passer l'Etat congolais pour un arbre fruitier, mais malencontreusement n'est pas toujours prêt à porter de fruits escomptés, à l'occurrence un Etat de droit où le droit aux soins de santé de bonne qualité est une jouissance effective.

En quoi est-il moins contradictoire de proclamer " Etat de droit... » une République qui, par la réforme des finances publiques, ne parvient pas encore à rendre effective la jouissance du droit aux soins de sa santé de bonne qualité et pour les gouvernants et pour les gouvernés?

De 1998 à 2013, il est constaté que les évolutions apportées au secteur de la santé, précisément au Ministère de la Santé Publique, décrivent les taux budget alloué à la santé et taux d'exécution respectivement, d'une manière contradictoire à l'édification d'un Etat de droit.

\begin{tabular}{|l|r|r|r|}
\hline Année & \multicolumn{3}{|c|}{ Budget Santé } \\
\hline & $\begin{array}{c}\text { Taux budget alloué } \\
\text { à la santé }\end{array}$ & $\begin{array}{c}\text { Taux d'exécution } \\
\text { budget Santé }\end{array}$ & $\begin{array}{c}\text { Taux d'exécution budget San- } \\
\text { té sur exécution global }\end{array}$ \\
\hline 1998 & $1,81 \%$ & $89 \%$ & $0,52 \%$ \\
\hline 1999 & $1,69 \%$ & $100 \%$ & $0,41 \%$ \\
\hline 2000 & $0,97 \%$ & $17,02 \%$ & $0,17 \%$ \\
\hline 2001 & $1,93 \%$ & $56,60 \%$ & $0,72 \%$ \\
\hline 2002 & $0,81 \%$ & $19,98 \%$ & $0,18 \%$ \\
\hline
\end{tabular}

24 J ABOLIA, op. cit, pp $105-395$.

25 Article $1^{\text {er }}$, al $1^{\text {er }}$ de la constitution du 18 février 2006. 


\begin{tabular}{|l|r|r|r|}
\hline Année & \multicolumn{3}{|c|}{ Budget Santé } \\
\hline & $\begin{array}{c}\text { Taux budget alloué } \\
\text { à la santé }\end{array}$ & $\begin{array}{c}\text { Taux d'exécution } \\
\text { budget Santé }\end{array}$ & $\begin{array}{c}\text { Taux d'exécution budget San- } \\
\text { té sur exécution global }\end{array}$ \\
\hline 2003 & $4,90 \%$ & $55,53 \%$ & $3,22 \%$ \\
\hline 2004 & $5,43 \%$ & $32,63 \%$ & $2,34 \%$ \\
\hline 2005 & $4,46 \%$ & $54,75 \%$ & $3,58 \%$ \\
\hline 2006 & $4,03 \%$ & $54,00 \%$ & $3,26 \%$ \\
\hline 2007 & $3,62 \%$ & $108,83 \%$ & $5,93 \%$ \\
\hline 2008 & $3,02 \%$ & $99,68 \%$ & $3,88 \%$ \\
\hline 2009 & $5,29 \%$ & $63,39 \%$ & $4,75 \%$ \\
\hline 2010 & $5,85 \%$ & $52,53 \%$ & $5,14 \%$ \\
\hline 2011 & $3,47 \%$ & $58,77 \%$ & $4,11 \%$ \\
\hline 2012 & $7,82 \%$ & $20,43 \%$ & \\
\hline 2013 & $4,57 \%$ & $15,64 \%$ & \\
\hline
\end{tabular}

Le constat est que de 2000 à 2013, le taux des budgets alloués au secteur de la santé n'ont même pas atteint $10 \%$ des budgets globaux de l'Etat; il va de même pour les taux d'exécution des budgets santé sur les exécutions globales. Ce qui est positif c'est l'évolution crescendo qui transparait à travers les taux d'exécutions budgets santé sur les exécutions globales. Mais cette évolution crescendo est consécutive au dépassement des dépenses au détriment des prévisions. Or il semble que le déséquilibre entre ressources et charges n'est profitable qu'en cas d'un budget d'investissement.

b. L'édification de l'Etat de droit à l'épreuve de l'aide internationale consacré à la santé en République démocratique du Congo

L'aide extérieur consacrée à la santé sous forme de projets constitue le mode de financement extérieur. Elle a certes augmenté depuis 2001, mais elle demeure en grande partie destinée à la lutte sélective contre la maladie. C'est le cas de tout le financement qui provient $\mathrm{du}$ fonds Mondial de lutte contre le Paludisme, le VIH/SIDA et la tuberculose et d'autres initiatives mondiales de santé. ${ }^{26}$

Le fait que ces ressources destinées à la lutte sélective contre la maladie aient été pratiquement les seules disponibles dans le secteur de la santé au lendemain de la redéfinition de la politique nationale de santé, a certainement contribué à la marginalisation de la stratégie basée sur les sons de santé primaires et de la zone de santé comme unité opérationnelle. ${ }^{27}$

26 Lire le plan National de développement sanitaire, (PNDS), 2011 - 2015, op. cit, pp.49-50.

27 Idem. 
On a donc assiste à la mise en place des services de santé basés sur la satisfaction des besoins des bailleurs en terme d'évolution des indications des programmes spécialisés en lieu et place des services de santé centrés sur l'homme et pour le droit de la population congolaise.

Plus l'homme est hoté du centre des préoccupations des services de santé, plus la protection des droits de la personne humaine devient un épiphénomène.

Cependant, il y a lieu de se convaincre qu'au delà de la Constitution qui sacralise la personne humaine en obligeant, outre, l'Etat de la respecter et de la protéger, ${ }^{28}$ la bonne volonté politique en faveur de la promotion et la protection des droit de la personne humaine se révèle cruellement lacunaire si la part du budget de l'Etat alloué à la santé n'est pas à la hauteur des défis, dont certains, tel que l'aide internationale à la santé, sont pris pour de solutions. Et pourtant cette aide est encore un problème à résoudre.

On note que l'aide destiné aux services de santé de base est restée constante alors que celle destinées à la lutte sélective contre la maladie a plus que doublé. ${ }^{29}$

La forte dépendance du financement de la santé vis-à-vis de l'extérieur à fortement influencé la structure du financement de la santé d'une manière défavorable. ${ }^{30}$

Il est à noter cependant que la plupart des financements internationaux allouées au secteur de la santé en R.D. Congo ont un caractère extrabudgétaire avec un faible alignement et peu d'harmonisation. ${ }^{31}$

Ils profitent plus à certains gouvernants qu'aux gouvernés ce qui rend difficile l'édification de l'Etat de droit tant attendu. Une telle situation rend favorable le développement du financement communautaire. On se demande si ce dernier ne pose pas de problème?

c. Les problèmes du financement communautaire et l'édification d'un Etat de droit en République Démocratique du Congo.

Au début des années 1990, avec la compression drastique des financements publics et l'arrêt presque complet de l'aide internationale à la suite de la rupture de la coopération structurelle avec les institutions de Bretton Woods, les ménages étaient amenés à supporter la quasi-totalité de la charge financière des services de santé.

En effet, ce mode de paiement direct par les ménages au lieu où sont administrés les soins, constitue un important obstacle financier à l'accès aux soins par les couches les plus pauvres de la population et a entrainé une baisse du taux d'utilisation des services curatifs passant de 0,60 contacts par habitant et par an pendant les années 80 à $0,5(0,07-0,42)$ contacts pendant les années 1990 à $2000 .^{32}$ Le paiement direct entre ainsi en conflit avec

28 Lire 1'Article 16, al. $1^{\text {er }}$ de la constitution du 18 février 2006.

29 Lire le plan National de développement sanitaire, p.50.

30 Idem.

31 Ibidem, p. 51.

32 Lire le Rapport de la Banque Mondiale 2005 pour le domaine de la Santé. 
l'une des caractéristiques des soins de santé primaires à savoir : l'équité en matière de soins. ${ }^{33}$

Deux tiers de patients en R.D. Congo ne recourent pas au système de santé pour obtenir des soins, soit parce que les services ne sont pas disponibles ou sont de mauvaise qualité quand ils existent, soit parce qu'ils n'ont pas de moyens (financiers) pour y accéder.

Selon une étude réalisée par l'Ecole de Santé Publique de l'Université de Kinshasa en 2003, parmi les membres de familles qui sont tombés malades, $30 \%$ sont allés dans un centre de santé public ou confessionnel, $40 \%$ ont pratiqué l'automédication, $21 \%$ n'ont reçu aucun traitement et $9 \%$ ont consulté un guérisseur traditionnel. ${ }^{34} \mathrm{Ceci}$ correspond à environ $70 \%$ des malades qui n'ont pas accès aux services de santé modernes.

Dans cette situation, l'édification de l'Etat de droit demeure encore un problème quoique la constitution a déjà proclamé que la RDC est un Etat de droit. ${ }^{35}$

A cet égard, l'Etat de droit ne doit pas seulement se développer dans le contexte de sa conception politique de limitation du pouvoir par opposition à un Etat de police comme nous semblons comprendre l'argumentation du Professeur KAMU KUNY. ${ }^{36}$

Il doit aussi et nécessairement être perçu avec acuité, comme un Etat dont le niveau de la promotion de la justice sociale implique un répondant très favorable au budget alloué à la santé. Rien de bon peut se faire pour accomplir ses devoirs citoyens lorsqu'on est pas en bonne santé.

Cette conception fait sans doute bon ménage avec les composantes du contenu minimum d'un Etat de droit pour qui la primauté est accordée à l'effectivité de jouissance à la bonne santé, à la suite d'un accès pour tous, aux bonnes prestations des services des soins de santé de base. Pour y parvenir faut-il nécessairement impliquer le financement privé?

d. L'édification de l'Etat de droit à l'épreuve du financement privé consacré au secteur de la santé en République Démocratique du Congo.

Le secteur privé lucratif et non lucratif a toujours été un partenaire traditionnel de l'Etat dans l'offre des soins. Sa contribution est sans doute importante, même si elle n'est pas bien connue. A titre d'exemple, le privé lucratif, non lucratif et les entreprises détiennent $40 \%$ d'hôpital général de référence des zones de santé du pays. ${ }^{37}$ Voilà combien la population est abandonnée entre les mains du privé.

Le secteur privé à but lucratif s'est considérablement développé dans les villes bien que la hauteur de sa contribution au financement de la santé reste méconnue. Et pourtant, le financement des services de santé par les entreprises, particulièrement les entreprises

33 Lire le plan National de développement sanitaire, (PNDS), 2011 - 2015, op. cit, pp.51-52.

34 Lire le plan National de développement sanitaire, (PNDS) 2011 - 2015, op. cit., pp.51-52.

35 Article $1^{\text {er }}$, al $1^{\text {er }}$ de la constitution du 18 février 2006.

36 A. KAMUKUNY MUKINAY, Droit constitutionnel congolais, Editions Universitaires Africaines, Kinshasa, 2011, p.40.

37 Lire le plan National de développement sanitaire, (PNDS), 2011 - 2015, op. cit, pp.53. 
publiques, demeure encore important. En 1989, la contribution des entreprises paraétatiques au financement du secteur était estimée à 142 millions de dollars américains. ${ }^{38}$

De façon générale, les dépenses de santé en RD Congo restent encore très faibles par rapport aux besoins. Elles sont parmi les plus faibles des pays à produit intérieur brut inférieur à 1200 dollars américains. ${ }^{39}$

Le défaut de ramener le budget alloué au secteur de la santé à la hauteur des défis qui guettent ce secteur, notamment l'inaccessibilité aux soins de santé de bonne qualité, par manque de moyens financiers, des services disponibles et des personnels qualifiés, constitue une violation faite à la sacralité de la personne humaine.

Ainsi, violer l'alinéa premier de l'article 16 de la constitution n'est pas moins grave que la violation d'autre dispositions constitutionnelles.

\section{LES CARACTERISTIQUES DE L'EVOLUTION D'EXECUTION DES DEPENSES DU SECTEUR DE LA SANTE ET L'EDIFICATION DE L'ETAT DE DROIT.}

Nous avons décriée, dans la première partie, la minoration du montant qu'il fallait allouer au secteur de la santé. Son augmentation, à travers les évolutions apportées à ce secteur, par la reforme des finances publiques, demeure toujours loin de répondre significativement aux différents besoins y relatifs.

Il se pose, en outre, plusieurs problèmes qui caractérisent l'exécution des dépenses. Ces derniers s'ajoutent à celui du maigre enveloppe budgétaire alloué à la santé.

Les caractéristiques de l'exécution des dépenses publiques du secteur de la santé ne s'écarte pas à la situation globale d'exécution des dépenses publiques de l'Etat. C'est ce qui nous convient de développer ici.

Les dépenses publiques, l'on sait bien qu'elles comportent quatre phases successives : l'engagement, la liquidation, l'ordonnancement et le paiement. ${ }^{40}$ Mais il existe une pluralité des procédures. Cela est principalement inhérent à la diversité de la nature des dépenses auxquelles le Trésor public doit constamment faire face et se manifeste plus particulièrement au stade de l'engagement et de la liquidation. ${ }^{41}$ Le non respect récurent de ces procédures caractérisent l'exécution des dépenses de la santé et amenuise subséquemment la jouissance du droit aux soins de santé de bonne qualité.

38 Idem.

39 Ibidem.

40 J.M. ABOLIA, op. cit., p. 139.

41 R. UMBA - DI - NDANGI, Finances publiques, commentaires de principes, procédures, Pratiques des origines à nos jours en République Démocratique du Congo, Kinshasa, B.E.C.I.F, 2006, p.85. 
a. Le non respect des procédures d'exécution des dépenses publiques de la santé et l'édification d' un Etat de droit

Le non respect des procédures d'exécution des dépenses publiques du secteur de la santé se constate par : la violation permanente des procédures d'exécution des dépenses publiques en général et la multiplicité des centres d'ordonnancement.

aa. La violation permanente des procédures d'exécution des dépenses publiques en général.

L'exécution des dépenses publiques en général et du secteur de la santé en particulier est caractérisée par le recours aux procédures exceptionnelles ou tout simplement irrégulières pour mouvementer le compte général du Trésor. ${ }^{42}$

Le règlement sur la comptabilité publique indique la procédure à suivre, c'est à dire le respect de la succession régulière de quatre phases ci-haut citées. Le bon d'engagement accompagné de pièces justificatives est le document de base qui permet d'enclencher ces différentes phases. ${ }^{43}$

Il convient de ne pas se perdre de vue que l'application stricte de cette procédure devrait logiquement conduire à une exécution des dépenses dans les limites prévues par la loi budgétaire.

En pratique, il est constaté que les deux premières phases d'engagement ont très souvent été occultées. Cette violation des procédures réglementaires a fait que les dépenses controlées au niveau du Ministère du budget sont estimées à environ $20 \%$ des dépenses budgétaires. ${ }^{44}$

Pour ce qui est des titres permettant de mouvementer le compte général du trésor, des décaissements importants sont opérées au moyen des titres inappropriés ou tout simplement suite à des instructions verbales. Par exemple, dans un rapport portant sur les opérations ayant affecté ce compte durant l'exercice budgétaire 1994, la cour des compte avait relevé que $27,25 \%$, soit près du tiers de l'ensemble des décaissements, ont été effectués par un recours soit à des titres de paiement inappropriés (de simples lettres) soit sans aucun titre, c'est à dire sur instruction verbale.

Cette façon de procéder ne permet pas une imputation correcte des dépenses eu égard aux articles budgétaires et aboutit au détournement des crédits de leur destination, voire à des dépassements massifs des crédits, en faveur des postes généralement non prévus au détriment de ceux qui sont régulièrement inscrits au budget. ${ }^{45}$

42 MABI MULUMBA, les dérivés d'une gestion prédatrice, le cas du Zaïre devenu République Démocratique du Congo, Editions CEDI, Kinshasa, 2002, p.56.

43 MABI MULUMBA, les dérivés d'une gestion prédatrice, le cas du Zaïre devenu République Démocratique du Congo, Editions CEDI, Kinshasa, 2002, p.56.

44 MABI MULUMBA, op. cit., p. 56.

45 MABI MULUMBA, op. cit., p. 56. 
Une telle situation ne permet pas à la population de jouir de soins de santé de bonne qualité. Car les services de santé publique qui est débiteur du droit aux soins de santé de bonne qualité subissent les conséquences fâcheuses dudit détournement. L'édification d'un Etat de droit doit cependant se faire sans droit aux soins de santé de bonne qualité.

bb. La multiplicité des centres d'ordonnancement

Toujours le même rapport de la cour des comptes note qu'en 1994, en violation de la loi financière, la Banque Centrale s'est érigée en un centre d'ordonnancement en faisant débiter le compte général du Trésor aux moyens des « Notes du Gouverneur », des cartons de trésorerie et même suite aux ordres verbaux. Par cette procédure irrégulière, la Banque Centrale a mouvementé le compte du Trésor pour 21,98\% de l'ensemble des décaissements pour l'exécution budgétaires de l'année. Ce faisant elle a violé la loi financière qui dispose en son article 33, que le ministre des Finances est le seul ordonnateur général du budget de l'Etat. ${ }^{46}$

Cette pratique constitue une dérive permanente des procédures administratives des décaissements du compte du Trésor. En effet, elle ne s'observe pas uniquement durant les années récentes. Elle a été à la base d'une dissipation de près de 400 millions de dollars en 1988 par rapport à 1987 au titre des dépenses en devises du Gouvernement hors dette extérieure. ${ }^{47}$ Cette situation n'a pas épargner le bon fonctionnement escompté du secteur de la santé. Les dépenses de ce secteur se sont retrouvées amenuisées du point de vue taux d'exécution.

Dans l'hypothèse d'une certaine cohérence dans l'action gouvernementale, cette somme de 400 millions de dollars aurait pu assurer largement la réhabilitation et l'approvisionnement des infrastructures sanitaires. Ce qui est troublant est que la banque centrale n'indique pas la destination de cette somme qui aurait représenté environ $30 \%$ des dépenses budgétaires de 1988 .

b. Quelques performances et prospective de l'évolution d'exécution des dépenses publiques du secteur de la santé.

Les caractéristiques de l'évolution d'exécution des dépenses publiques du secteur de la santé se révèle défavorables, bien sûr, à l'édification d'un Etat de droit non dépourvu du droit à la jouissance des soins de santé de bonne qualité. Mais il ya lieu de déceler quelques performances de bonne espérance. Le plan national de développement sanitaire constitue sans doute un effort considérable pour ces quelques performances, dans une prospective certaine et une bonnes espérances.

46 Idem.

47 Ibidem. 
Le cadre macroéconomiques du Plan national 2011 - 2015 qui nous a servi de référence est basé sur des documents officiels du Ministère des Finances, du Ministère du Plan, du Ministère du Budget et des institutions de Bretton Woods.

En particulier, l'analyse a pris en compte les résultats d'un précédent exercice du Ministère de la santé publique sur l'évolution observée des agrégats économiques nationaux et leur évolution probable sur les 5 ans du plan national de développement sanitaire.

Parmi les documents principaux, on peut citer la revue des Dépenses Publiques 19 de 2008 et le tableau des indicateurs économiques et financier, 2007 - 2014 élaboré par le Ministère des Finances et les services du Fonds Monétaire International. Il apparait qu'en dépit d'un environnement économique international morose, voire de récession, et son impact négatif sur la croissance du pays en 2009, la tendance au relèvement de l'économie nationale s'affirme et devrait se renforcer durant les prochaines années. ${ }^{48}$

En effet, d'un niveau de croissance de 6,3\% et 6,2\% respectivement en 2007 et 2008, le taux estimé pour 2009 est tombé à $2,7 \%$. Il devrait remonter à un niveau moyen de $6,92 \%$ sur la période 2010 à 2014 .

Le scénario de projection des ressources prévisibles pour le secteur de la santé sur la période du plan national de développement sanitaire, se base sur un taux de croissance réel de l'économie qui se situera autour d'une moyenne de 7,44\% (2011 - 2015) et sur la moyenne de $32,72 \%$ du ratio des dépenses publiques en $\%$ du produit intérieur brut pour la même période. Si l'on adopte ces deux agrégats et que l'effort actuel est maintenu, la moyenne du ration des dépenses publiques totales de santé sera de 7,9\% sur la période du plan, et représentera 9,3\% en $2015 .{ }^{49}$ Dans ce cas, le gap vis-à-vis de l'objectif de $15 \%{ }^{50}$ ne sera plus que de $6 \%$.

En effet, le financement maximal permettra, dans cette perspective, d'atteindre 10,3\% du budget de l'Etat alloué à la santé en 2015, tout en conduisant une politique minimale de réduction de la contribution des ménages aux dépenses du secteur de la santé. Les dépenses totales de santé par tête d'habitant atteindraient 35,07 USD en 2015. ${ }^{51}$

En définitive, quoique les caractéristiques de l'évolution d'exécution du budget de l'Etat, dans le secteur de la santé, paraissent défavorables à la jouissance des soins de santé de bonne qualité, il est moins légitime que le pessimisme se développe indéfinitivement devant les performances certaines que nous venons de dépeindre. Car pendant la période du plan, les dépenses totales de santé par habitant et par an passeront de 24,17 USD en 2011 à 32,73 UDS en 2015 soit un accroissement de 35\% dans l'hypothèse normale de projection des ressources prévisibles pour le secteur alors qu'avec l'hypothèse optimiste les dépenses totales de santé par habitant et par an passeront de 24,55 USD en 2011 à 35,07 USD en

48 Lire le plan National de développement sanitaire, (PNDS), 2011 - 2015, op. cit, p.92.

49 Idem, p.93.

50 Engagement que la République Démocratique du Congo avait pris à Abuja en 2001 sur l'augmentation du budget de la Santé pour 2015.

51 Lire le plan National de développement sanitaire, (PNDS), 2011 - 2015, op. cit, p.93. 
2015. ${ }^{52}$ Ce qui permet d'espérer que l'édification de l'Etat de droit en République Démocratique du Congo intègre de manière étapiste le droit aux soins de santé de bonne qualité quoique timidement.

\section{CONCLUSION}

Les hypothèses de recherche formulées à l'introduction de la présente étude se sont révélées confirmées, chiffres à l'appui, à la suite de la confrontation aux faits. Le budget de l'Etat consacré à la santé reste faible et largement inférieur aux engagements des chefs d'Etat à Abuja (15\%). Dans la plupart des cas, il est inférieur à 5\% du budget global de l'Etat et son taux de décaissement est en moyenne de 70\% (exercice 2008 et 2009). Donc de 1998 à 2013, son affectation ne tient pas compte des priorités du secteur où doit se concrétiser le droit à la vie, mieux le droit à une vie de bonne qualité humaine appuyé par le droit aux soins de santé de bonne qualité.

L'aide internationale consacrée à la santé demeure fragmentée. Cette fragmentation de l'aide dans un contexte marqué par le désengagement de l'Etat a conduit à celle des prestations mettant ainsi à rude épreuve le caractère global, continu, intégré des soins de santé primaire dans les zones de santé.

Une telle situation fait que l'édification de l'Etat de droit en République Démocratique du Congo, intègre difficilement et avec parcimonie le droit à la jouissance effective des soins de santé de bonne qualité. Toutes fois, les efforts se déploient à travers des performances quoique chétives, mais non négligeables, indiquant ainsi une évolution améliorative, à travers le plan national du développement sanitaire 2011 - 2015, pour aboutir à cette jouissance tant attendue.

\section{BIBLIOGRAPHIE}

1. Textes légaux et réglementaires

Constitution de la République Démocratique du Congo du 18 février 2006, Journal Officiel de la République Démocratique du Congo, $47^{\mathrm{e}}$ année, numéro spécial

Loi n ${ }^{\circ} 005-2002$ du 7 mai 2002 relative à la constitution, organisation et fonctionnement de la Banque

Centrale du Congo, les codes Larciers de la R.D. Congo, Tome III, Vol.2

Loi financier $\mathrm{n}^{\circ} 83-003$ du 23 février 1983 telle que modifiée et complétée par l'ordonnance loi n

๑87-004 du 10 janvier 1998, Les codes Larciers de la R.D. Congo, Tome VI, Vol.1

Loi n`76 - 021 du 16 septembre 1976 portant création de l'office de gestion de dettes publiques, les

codes Larciers de la R.D. Congo, Tome VI, Vol.1

Loi ${ }^{\circ} 76-20$ du 16 juillet 1976 portant normalisation de la comptabilité au (Congo), Les codes

Larciers de la R.D. Congo, Tome III, Vol.2

Ordonnance $\mathrm{n}^{\circ} 78-032$ du 30 septembre 1978 portant règlement de la comptabilité des zones. 
Arrêté Royal du 18 décembre 1951 portant règlement sur la comptabilité publique

Circulaire $n^{\circ} 001 / \mathrm{CAB} / \mathrm{MIN} / \mathrm{BUDGET} / 2006$ du 23/02/2006 contenant les instructions relatives à l'exécution du budget de l'Etat pour l'exercice 2006 Ministère du Budget/R.D. Congo

Vade - mecum des pièces justificatives relatives à l'exécution des dépenses publiques du 18/03/2006 du ministère du budget.

\section{Ouvrages}

ABOLIA, J.M., Finances Publiques et comptabilité de l'Etat en République Démocratique du Congo, Principes, pratique et perspectives, B.E.SI.F, Kinshasa, 2005

BAKANDEJA wa MPUNGU, Les finances publiques. Pour une meilleure gouvernance économique et financière en République Démocratique du Congo, Afrique Editions, 2006.

Banque Mondiale, Pour une meilleure santé en Afrique, les leçons de l'expérience, la série « développement à l'œuvre », Banque Mondiale, D.C. Washington.

KAMUKUNY MUKINAY, Droit constitutionnel congolais, Editions Universitaires Africaines, Kinshasa, 2011.

KIBUEY MULAMBU, le Budget de l'Etat - Règles et Techniques de gestion, ECA-IRES, 1 ère éd. 1988.

MABI MULUMBA, LES dérives d'une gestion prédatrice, le cas du Zaïre devenu République Démocratique du Congo, Editions CEI, Kinshasa, 2002.

Observations de la cour des comptes relatives au projet de loi portant Arrêt des comptes te règlement définitif du budget de l'Etat pour l'exercice 2003, Journal officiel de la RDC, $46^{\mathrm{e}}$ année, numéro spécial.

Reddition des comptes 1987,1988, 2001 et 2002, Cahier des observations de la Cour des comptes relatives au projet de loi portant Arrêt des comptes et Règlement définitif du budget de l'Etat pour ces exercices, Journal officiel Spécial Cour des comptes du 28 janvier 2005, 46 année.

UMBA - DI- NDANGI, Finances Publiques, commentaires de principes, procédures, pratiques des origines à nos jours, en RDC, Kinshasa, B.E.C.I.F, 2006 